\title{
Overall Survival in Patients with Mucinous Carcinoma of Breast: A Population-Based Study
}

\author{
Tingting $\mathrm{Hu}\left(\mathbb{D}^{\prime}\right.$ \\ Juanjuan Huang ${ }^{2}$ \\ Kun Fang ${ }^{3}$ \\ 'Department of Chemoradiation \\ Oncology, The First Affiliated Hospital of \\ Wenzhou Medical University, Wenzhou, \\ Zhejiang, 325000, People's Republic of \\ China; ${ }^{2}$ Wenzhou Medical University, \\ Wenzhou, Zhejiang, 325000, People's \\ Republic of China; ${ }^{3}$ Department of \\ Surgery, Yinchuan Women and Children's \\ Hospital, Yinchuan, Ningxia, 75000I, \\ People's Republic of China
}

Correspondence: Kun Fang Department of Surgery, Yinchuan Women and Children's Hospital, No. 56, Wenhua Street, Yinchuan, Ningxia, 75000I,

People's Republic of China

Email k99ft|@I63.com
Purpose: Mucinous carcinoma of the breast (MCB) is a rare malignant tumour. Therefore, it is urgent to establish a survival prediction model for MCB patients.

Methods: Clinicopathological and follow-up data of MCB patients diagnosed between 2010 and 2015 were retrieved from the Surveillance, Epidemiology, and End Result (SEER) database. The significant factors were screened out and generated Kaplan-Meier (K-M) curves for each prognostic factor. Additionally, these factors were then utilized to build a nomogram for predicting 3-, 4-, and 5-year overall survival (OS) of MCB patients. The nomogram was evaluated using calibration curves, receiver operating characteristic (ROC) curves and decision curve analysis (DCA).

Results: Moreover, a total of $4326 \mathrm{MCB}$ patients were retrieved. Age, American Joint Committee on Cancer (AJCC) stage, surgery, radiotherapy and bone metastasis were identified as independently prognosis factors for OS. The corresponding areas under the ROC curves (AUCs) of the nomogram at 3,4 and 5 years in the training and validation set were $0.770,0.788,0.805,0.778,0.797$, and 0.802 , respectively. The calibration curves and DCA revealed that the prediction model had an excellent performance. Finally, the risk stratification system confirmed that the powerful role of the nomogram in distinguishing results and risk stratification.

Conclusion: Briefly, the nomogram incorporating various clinicopathological indicators was established for MCB patients and may facilitate clinical decision-making.

Keywords: mucinous carcinoma of the breast, breast cancer, prognosis, nomogram

\section{Introduction}

According to the report, the breast cancer-specific deaths in 2018 accounted for approximately $15 \%$ of female cancer deaths. ${ }^{1}$ Mucinous carcinoma of the breast (MCB) is a rare indolent tumour and accounts for $2-4 \%$ of all breast cancers. ${ }^{2,3}$ It is common in perimenopausal and postmenopausal women, ${ }^{4}$ and it is characterized by tumour cells floating in mucin. ${ }^{5} \mathrm{MCB}$ is distinct from other breast cancer subtypes and is usually associated with a favourable outcome, low recurrence rate, and low incidence of lymph node metastasis. ${ }^{6,7}$

MCB is a form of infiltrating ductal carcinoma (IDC). Fortunately, it showed a better prognosis than other types of IDC and often required only local treatment. ${ }^{8}$ The morphology and prognosis of tumours with abundant extracellular mucins can be significantly different from other types of breast cancer. ${ }^{9}$ In addition, the prognosis of patients with different histologic types is significantly different. ${ }^{10}$ Therefore, identifying patients with indolent and low-risk tumours is crucial. It is also highly beneficial in the clinical management of $\mathrm{MCB}$ and optimizing the 
delivery of limited health resources. The traditional American Joint Committee on Cancer (AJCC) stage system cannot effectively predict the prognosis of patients. ${ }^{11}$ A robust prognostic biomarker and model is urgently needed. In previous studies, multiple biomarkers and prognostic variables were identified for MCB patients, including age, tumour size, lymph node status, and estrogen receptor (ER) status. ${ }^{12,13}$ However, regrettable is that no prognostic prediction model was established for MCB patients. Therefore, developing a nomogram incorporating other prognostic variables is needed.

The nomogram is a convenient prediction tool that accurately predicts individual prognosis and has been established to assess the outcome of several cancers. ${ }^{14}$ Therefore, we aimed to construct a prognostic model to predict the prognosis of patients by analyzing a population-based MCB cohort.

\section{Materials and Methods}

\section{Patients Selection}

Patients' data were acquired using the SEER*Stat 8.3.6. Female patients diagnosed with MCB between 2010 and 2015 were included. The exclusion criteria were as follows: (1) MCB is not the first primary tumour; (2) died but the cause of death is unclear; (3) unknown information, including age, tumour size, race, grade, AJCC TNM stage, surgery, radiotherapy, chemotherapy, brain metastasis, bone metastasis, liver metastasis, lung metastasis, estrogen receptor (ER) status, progesterone receptor (PR) status, human epidermal growth factor 2-neu (HER2) status and marital status. Patients meeting the screening criteria were randomly assigned to a training set $(70 \%)$ and a validation set $(30 \%)$. The nomogram was developed in the training set and validated in the validation set.

\section{Data Collection}

Variables were categorized based on demographic, cancer, treatment and metastatic data. Demographic variables include age, race, and marital status. The cancer characteristics included tumour size, grade, AJCC TNM stage, ER status, PR status, and HER2 status. Treatment characteristics included surgery, radiotherapy and chemotherapy. Metastatic data included brain metastasis, bone metastasis, liver metastasis, and lung metastasis. In the SEER database, age and tumour size were recorded as continuous variables. However, in our study, the X-tile software was utilized to confirm the optimal cutoff values of these two variables. ${ }^{15}$ The best cutoff values of age were 72 - and 82 years, and the best cutoff values of tumour size were 13 and $27 \mathrm{~mm}$.

\section{Statistical Analysis}

As previously mentioned, by using the X-tile software, the optimal cutoff values of age and tumour size were evaluated. ${ }^{15}$ Using multivariate Cox analyses to screen prognostic factors and a prognostic nomogram was built further. Meanwhile, the time-dependent receiver operating characteristic (ROC) curves of the prognostic nomogram were generated. ${ }^{16}$ The areas under the curves (AUCs) were used to evaluate the discriminability of the nomogram. Moreover, the consistency was estimated using calibration curves. Furthermore, the range of threshold probabilities and the magnitude of benefit was identified by DCA. Finally, patients were roughly classified into three risk groups, respectively. The value of the nomogram for predicting prognosis was verified by survival curve and Log rank test. The above statistical methods were performed using SPSS 25.0 (IBM) and R software (version 3.6.1). $P$ value $<0.05$ (two-sided) was considered as a statistically significant cutoff value.

\section{Results}

\section{Patients Baseline Characteristics}

The baseline information of $4326 \mathrm{MCB}$ patients is listed in Table 1. For all patients, 3260 (75.4\%) were White. The majority of the grade is I-II (96.1\%). Although bone metastases were the most likely to occur, it only accounts for $0.7 \%$ of cases. ER-positive accounted for $98.9 \%$, PRpositive accounted for $92.1 \%$, and HER2-negative accounted for $94.6 \%$. In addition, most MCB patients underwent surgery.

\section{Screening Prognostic Factors for MCB Patients}

The univariate Cox analysis was used to screen prognostic factors, and the results showed that age, tumour size, AJCC TNM stage, surgery, radiotherapy, chemotherapy, bone metastasis, brain metastasis, liver metastasis, lung metastasis, and marital status were OS-related factors (Table 2). Then, all related factors were incorporated into the multivariate Cox analysis, and age, AJCC stage, surgery, radiotherapy and bone metastasis were determined as independent related factors (Table 2). Then, the K-M survival curves were further plotted for each 
Table I Clinical and Pathological Features of Patients Diagnosed as MCB

\begin{tabular}{|c|c|c|c|}
\hline Characteristics & Total Set $(n=4326)$ & Training Set $(n=3030)$ & Validation Set $(n=\mid 296)$ \\
\hline \multicolumn{4}{|l|}{ Age, years } \\
\hline$<72$ & $2680(62.0 \%)$ & $1860(61.4 \%)$ & $820(63.3 \%)$ \\
\hline $72-82$ & $1179(27.3 \%)$ & $84 I(27.8 \%)$ & $338(26.1 \%)$ \\
\hline$>82$ & $467(10.8 \%)$ & $329(10.9 \%)$ & $138(10.6 \%)$ \\
\hline \multicolumn{4}{|l|}{ Tumor size, mm } \\
\hline$<13.0$ & $1559(36.0 \%)$ & $1099(36.3 \%)$ & 460 (35.5\%) \\
\hline $13.0-27.0$ & $1826(42.2 \%)$ & $1269(41.9 \%)$ & $557(43.0 \%)$ \\
\hline$>27.0$ & $94 \mathrm{I}(2 \mathrm{I} .8 \%)$ & $662(21.8 \%)$ & $279(21.5 \%)$ \\
\hline \multicolumn{4}{|l|}{ Race } \\
\hline White & $3260(75.4 \%)$ & $2319(76.5 \%)$ & $94 I(72.6 \%)$ \\
\hline Black & $535(\mid 2.4 \%)$ & $351(11.6 \%)$ & $184(\% \mid 4.2)$ \\
\hline Other & $531(12.3 \%)$ & $360(11.9 \%)$ & $17 \mid(13.2 \%)$ \\
\hline \multicolumn{4}{|l|}{ Grade } \\
\hline I & $2577(59.6 \%)$ & $1820(60.1 \%)$ & $757(58.4 \%)$ \\
\hline ॥ & $1579(36.5 \%)$ & $1087(35.9 \%)$ & $492(38.0 \%)$ \\
\hline III & $167(3.9 \%)$ & $120(4.0 \%)$ & $47(3.6 \%)$ \\
\hline IV & $3(0.1 \%)$ & $3(0.1 \%)$ & 0 \\
\hline \multicolumn{4}{|l|}{$\mathrm{AJCC}$} \\
\hline I & $2733(63.2 \%)$ & $1910(63.0 \%)$ & $823(63.5 \%)$ \\
\hline II & I388(32.1\%) & $975(32.2 \%)$ & $4 \mid 3(31.9 \%)$ \\
\hline III & $156(3.6 \%)$ & $110(3.6 \%)$ & $46(3.5 \%)$ \\
\hline IV & $49(1.1 \%)$ & $35(1.2 \%)$ & $14(1.1 \%)$ \\
\hline \multicolumn{4}{|l|}{ T stage } \\
\hline $\mathrm{TI}$ & $1805(64.8 \%)$ & $1955(64.5 \%)$ & $85,065.6(\%)$ \\
\hline $\mathrm{T} 2$ & $1242(28.7 \%)$ & $87 \mathrm{I}(28.7 \%)$ & $37 \mid(28.6 \%)$ \\
\hline $\mathrm{T} 3$ & $220(5.1 \%)$ & $163(5.4 \%)$ & 57 (4.4\%) \\
\hline $\mathrm{T} 4$ & $59(1.4 \%)$ & $4 I(I .4 \%)$ & $18(1.4 \%)$ \\
\hline \multicolumn{4}{|l|}{$N$ stage } \\
\hline No & $3908(90.3 \%)$ & 2750 (90.8\%) & $1158(89.4 \%)$ \\
\hline $\mathrm{NI}$ & $339(7.8 \%)$ & $227(7.5 \%)$ & II2(8.6\%) \\
\hline N2 & $53(1.2 \%)$ & $33(1.1 \%)$ & $20(1.5 \%)$ \\
\hline N3 & $26(0.6 \%)$ & $20(0.7 \%)$ & $6(0.5 \%)$ \\
\hline \multicolumn{4}{|l|}{ M stage } \\
\hline Mo & $4277(98.9 \%)$ & 2995(98.8\%) & $1282(98.9 \%)$ \\
\hline MI & $49(1.1 \%)$ & $35(1.2 \%)$ & I4(I.1\%) \\
\hline \multicolumn{4}{|l|}{ Surgery } \\
\hline No & 149(3.4\%) & $108(3.6 \%)$ & $4 I(3.2 \%)$ \\
\hline Yes & $4177(96.6 \%)$ & $2922(96.4 \%)$ & $1255(96.8 \%)$ \\
\hline \multicolumn{4}{|l|}{ Radiotherapy } \\
\hline No & $2085(48.2 \%)$ & | $443(47.6 \%)$ & $642(49.5 \%)$ \\
\hline Yes & $224 I(5 I .8 \%)$ & I587(52.4\%) & $65,450.5(\%)$ \\
\hline \multicolumn{4}{|l|}{ Chemotherapy } \\
\hline No & $3749(86.7 \%)$ & $2637(87.0 \%)$ & II I,285.8(\%) \\
\hline Yes & $577(13.3 \%)$ & $393(13.0 \%)$ & I84(|4.2\%) \\
\hline \multicolumn{4}{|l|}{ Bone metastasis } \\
\hline No & 4295(99.3\%) & 3008 (99.3\%) & $1287(99.3 \%)$ \\
\hline Yes & $3 \mathrm{I}(0.7 \%)$ & $22(0.7 \%)$ & $9(0.7 \%)$ \\
\hline \multicolumn{4}{|l|}{ Brain metastasis } \\
\hline No & $432 \mathrm{I}(99.9 \%)$ & $3026(99.9 \%)$ & 1295(99.9\%) \\
\hline Yes & $5(0.1 \%)$ & $4(0.1 \%)$ & $\mathrm{I}(0.1 \%)$ \\
\hline
\end{tabular}


Table I (Continued).

\begin{tabular}{|c|c|c|c|}
\hline Characteristics & Total Set $(n=4326)$ & Training Set $(n=3030)$ & Validation Set $(n=1296)$ \\
\hline \multicolumn{4}{|l|}{ Liver metastasis } \\
\hline No & $4316(99.8 \%)$ & $3023(99.8 \%)$ & $1293(99.8 \%)$ \\
\hline Yes & $10(0.2 \%)$ & $7(0.2 \%)$ & $3(0.2 \%)$ \\
\hline \multicolumn{4}{|l|}{ Lung metastasis } \\
\hline No & $4303(99.5 \%)$ & $3015(99.5 \%)$ & $1288(99.4 \%)$ \\
\hline Yes & $23(0.5 \%)$ & $15(0.5 \%)$ & $8(0.6 \%)$ \\
\hline \multicolumn{4}{|l|}{ ER } \\
\hline Negative & $49(1.1 \%)$ & $34(1.1 \%)$ & $15(1.2 \%)$ \\
\hline Positive & $4277(98.9 \%)$ & $2996(98.8 \%)$ & $|28|(98.8 \%)$ \\
\hline \multicolumn{4}{|l|}{ PR } \\
\hline Negative & $34 I(7.9 \%)$ & $237(7.8 \%)$ & $104(8.0 \%)$ \\
\hline Positive & $3985(92.1 \%)$ & $2793(92.2 \%)$ & $1192(92.0 \%)$ \\
\hline \multicolumn{4}{|l|}{ HER2 } \\
\hline Negative & $4094(94.6 \%)$ & $2862(94.5 \%)$ & $1232(95.1 \%)$ \\
\hline Positive & $232(5.4 \%)$ & $168(5.4 \%)$ & $64(4.9 \%)$ \\
\hline \multicolumn{4}{|l|}{ Marital status } \\
\hline No & $697(16.1 \%)$ & $475(15.7 \%)$ & $222(17.1 \%)$ \\
\hline Yes & $3629(83.9 \%)$ & $2555(84.3 \%)$ & $1074(82.9 \%)$ \\
\hline
\end{tabular}

Abbreviations: MCB, mucinous carcinoma of the breast; AJCC, American Joint Committee on Cancer; ER, estrogen receptor; PR, progesterone receptor; HER2, human epidermal growth factor 2-neu.

independent prognostic factor and illustrated that nomograms had an excellent ability to distinguish (Figure 1).

\section{Construction and Validation of the Nomogram for OS}

By integrating the independent prognostic factors, the OS nomogram was demonstrated (Figure 2). The AUCs in training set at 3-, 4-, and 5-years were $0.770,0.788$, and 0.805 , respectively, and the corresponding AUCs were 0.778, 0.797, and 0.802 in the validation set (Figure 3). Additionally, the calibration curves indicated that the predicted outcome was closed to the observed outcome (Figure 4). The DCA curves displayed that the prediction model has satisfactory predictive performance (Figure 5).

\section{Comparison of Discrimination Between Nomogram and Independent Prognostic Factors}

To further show the superior discrimination of our nomogram in assessing the survival of $\mathrm{MCB}$, we also plotted the ROC curves of the nomogram and all independent prognostic factors. The results indicated that the AUCs of all prognostic factors alone were higher than 0.500 , which means that all individual factors can serve as a reliable prognostic factor. The AJCC stage has the largest AUCs, indicating that the AJCC stage is the most effective single indicator. However, the AUCs of all prognostic factors were lower than those of nomograms (Figure 3). Generally, we concluded that the nomogram has more excellent discriminant ability than all the independent prognostic factors.

\section{Risk Stratifying for MCB Patients}

The total prognostic scores calculated by the nomogram was divided into three risk groups to predict prognosis. In the validation set, the cumulative probability of survival in three groups was further compared. According to the X-tile software, the optimal cutoff values for the total prognostic score were 5.90 and 11.17. Among them, $<5.90$ was the low-risk group, $>11.17$ was the high-risk group. The prognosis of the three risk groups is significantly different (Figure 6). These results all revealed that the nomogram was excellent to forecast the probability of survival in MCB.

\section{Discussion}

This study found that older age, higher AJCC stage, absence of surgery, absence of radiotherapy and bone metastasis were the main factors for the poor prognosis of MCB patients. We then constructed a prognostic nomogram incorporating corresponding independent prognostic 
Table 2 Univariate and Multivariate Cox Analysis in MCB Patients

\begin{tabular}{|c|c|c|c|c|}
\hline & & Univariate Analysis & & Multivariate Analysis \\
\hline & HR (95\% Cl) & $P$ value & HR (95\% Cl) & $P$ value \\
\hline \multicolumn{5}{|l|}{ Age, years } \\
\hline \multicolumn{5}{|l|}{$<72$} \\
\hline $72-82$ & $3.384(2.5 \mid 3-4.557)$ & $<0.001$ & $3.464(2.55 I-4.704)$ & $<0.001$ \\
\hline$>82$ & $8.926(6.595-12.081)$ & $<0.001$ & $7.766(5.644-10.685)$ & $<0.001$ \\
\hline \multicolumn{5}{|c|}{ Tumor size, mm } \\
\hline \multicolumn{5}{|c|}{$<13.0$} \\
\hline $13.0-27.0$ & $1.599(1.185-2.159)$ & 0.002 & & \\
\hline$>27.0$ & $2.710(1.985-3.701)$ & $<0.001$ & & \\
\hline \multicolumn{5}{|l|}{ Race } \\
\hline \multicolumn{5}{|l|}{ White } \\
\hline Black & $0.573(0.303-1.082)$ & 0.086 & & \\
\hline Other & $1.358(0.906-2.036)$ & 0.138 & & \\
\hline \multicolumn{5}{|l|}{ Grade } \\
\hline \multicolumn{5}{|l|}{ I } \\
\hline II & $1.023(0.797-1.313)$ & 0.857 & & \\
\hline III & $1.610(0.964-2.690)$ & 0.069 & & \\
\hline IV & $2.348(0.329-16.780)$ & 0.395 & & \\
\hline \multicolumn{5}{|l|}{ AJCC } \\
\hline \multicolumn{5}{|l|}{1} \\
\hline II & I.862(I.447-2.395) & $<0.001$ & $2.43 I(I .099-5.375)$ & 0.028 \\
\hline III & $2.26 I(I .345-3.80 I)$ & 0.002 & $2.169(0.820-5.740)$ & 0.119 \\
\hline IV & |8.298(| |.708-28.597) & $<0.001$ & $5.691(1.746-18.550)$ & 0.004 \\
\hline \multicolumn{5}{|l|}{ T stage } \\
\hline \multicolumn{5}{|l|}{ TI } \\
\hline $\mathrm{T} 2$ & $1.882(1.460-2.425)$ & $<0.001$ & $0.698(0.3|8-| .53 \mid)$ & 0.370 \\
\hline T3 & $2.287(1.493-3.503)$ & $<0.001$ & $0.657(0.290-1.484)$ & 0.312 \\
\hline $\mathrm{T} 4$ & $7.216(4.159-12.519)$ & $<0.001$ & $|.89|(0.705-5.07 \mid)$ & 0.205 \\
\hline \multicolumn{5}{|l|}{$\mathrm{N}$ stage } \\
\hline \multicolumn{5}{|l|}{ No } \\
\hline $\mathrm{NI}$ & $1.336(0.898-1.988)$ & 0.154 & & \\
\hline N2 & $2.094(0.864-5.079)$ & 0.102 & & \\
\hline N3 & $2.763(1.028-7.423)$ & 0.044 & & \\
\hline \multicolumn{5}{|l|}{ M stage } \\
\hline \multicolumn{5}{|l|}{ Mo } \\
\hline MI & 13.926(9.065-21.395) & $<0.001$ & & \\
\hline \multicolumn{5}{|l|}{ Surgery } \\
\hline \multicolumn{5}{|l|}{ No } \\
\hline Yes & $0.119(0.086-0.166)$ & $<0.001$ & $0.357(0.233-0.546)$ & $<0.001$ \\
\hline \multicolumn{5}{|l|}{ Radiotherapy } \\
\hline \multicolumn{5}{|l|}{ No } \\
\hline Yes & $0.388(0.388-0.302)$ & $<0.001$ & $0.616(0.470-0.807)$ & $<0.001$ \\
\hline \multicolumn{5}{|c|}{ Chemotherapy } \\
\hline \multicolumn{5}{|c|}{ No } \\
\hline Yes & $0.650(0.434-0.973)$ & 0.036 & & \\
\hline Bone metast & & & & \\
\hline No & & & & \\
\hline Yes & $15.610(9.537-25.550)$ & $<0.001$ & $3.490(1.318-9.238)$ & 0.012 \\
\hline
\end{tabular}

(Continued) 
Table 2 (Continued).

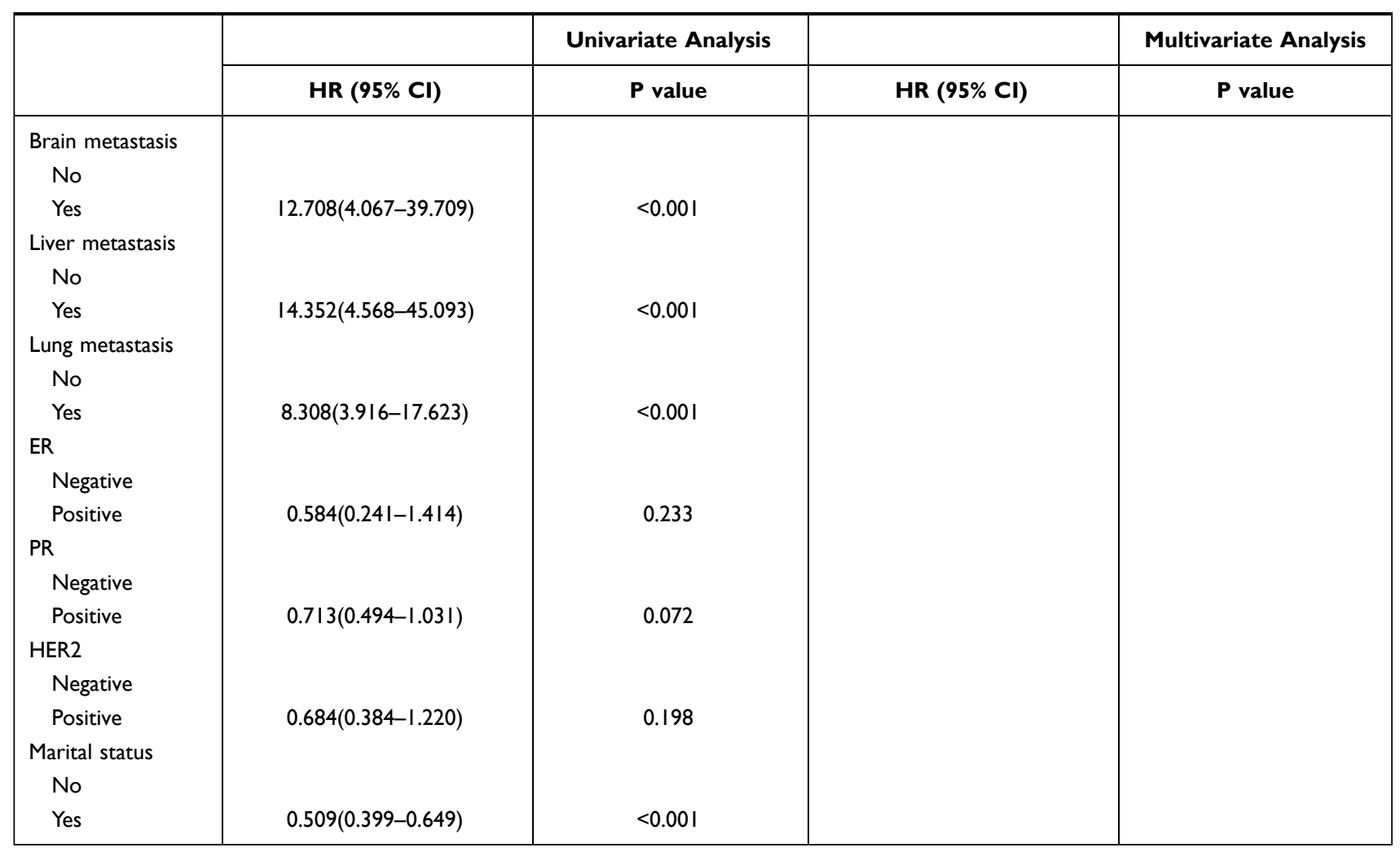

Abbreviations: MCB, mucinous carcinoma of the breast; HR, hazard ratio; Cl, confidence interval; AJCC, American Joint Committee on Cancer; ER, estrogen receptor; PR, progesterone receptor; HER2, human epidermal growth factor 2-neu.

clinical factors to predict OS better. The nomogram performed well in predicting survival in MCB patients. More importantly, the nomogram-based risk stratification systems were constructed to guide clinicians in decision making and disease monitoring.

In our research, age and the $\mathrm{AJCC}$ stage contribute the most to the prognosis. MCB is a disease of older women. The average age of patients is significantly older than those of other histological subtypes. ${ }^{17}$ Consistent with our results, age was strongly associated with the prognosis of $\mathrm{MCB}$ patients in a retrospective study. ${ }^{13}$ This may be because the poor prognosis of elderly patients was not only related to the clinical course, but also related to comorbidities. ${ }^{18}$ In addition, taking into account their poor functional status, only less active treatment was performed, resulting in a relatively poor prognosis. ${ }^{19}$ AJCC stage is a widely accepted prognostic factor for cancer patients. It considers the primary tumour, local metastasis, and distant metastasis. Several studies have shown that by integrating the AJCC stage and other clinical prognostic indicators, the accuracy of predicting the prognosis of cancer patients can be significantly improved. In this study, the nomogram incorporates the AJCC stage and other prognostic clinicopathological parameters. We have observed that the AUCs of the nomogram was higher after integrating other indicators.

Although the general prognosis of MCB patients was excellent, unfortunately, MCB also occasionally undergoes local recurrence or distant metastasis. ${ }^{20}$ We found that patients with distant metastases had a lower survival rate. Among them, bone metastasis is pivotal because it is the leading cause of death in patients with advanced breast cancer. $^{21,22} \mathrm{MCB}$ is distinct from breast cancer. It has specific heterogeneity, such as high expression of hormone receptors and low expression of human epidermal growth factor receptor 2 (EGFR2/HER2). ${ }^{23,24}$ Moreover, several studies have revealed that low-grade and ER-positive tumours are more prone to bone metastasis. ${ }^{25-27}$ Therefore, it should be considered in the treatment of advanced patients to improve the survival rate of these patients.

Regarding treatment factors, surgery and radiotherapy were related to the prognosis of $\mathrm{MCB}$ patients. $\mathrm{MCB}$ is 
A

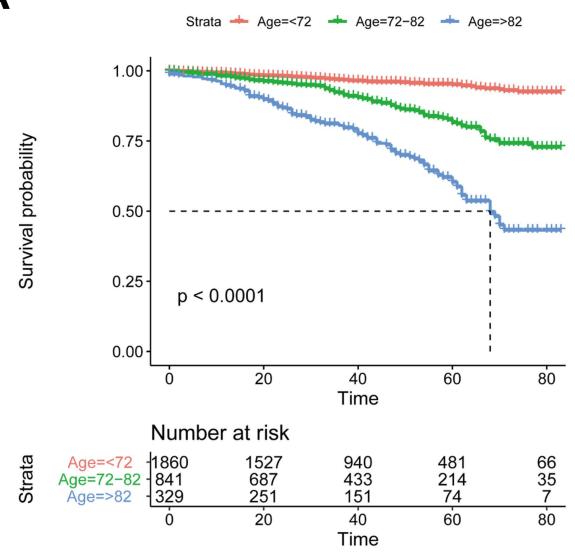

C
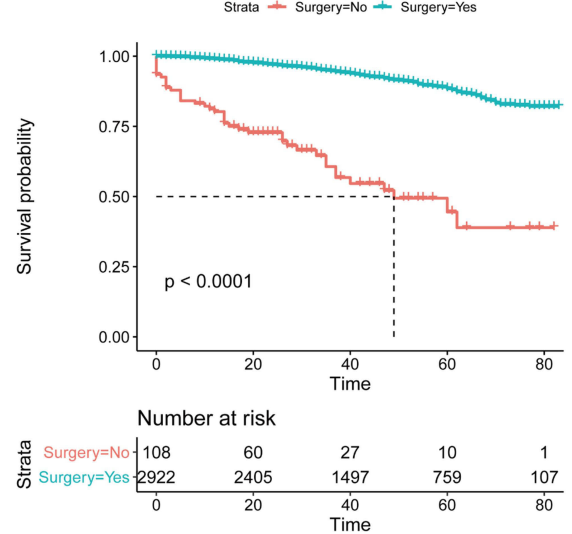

E

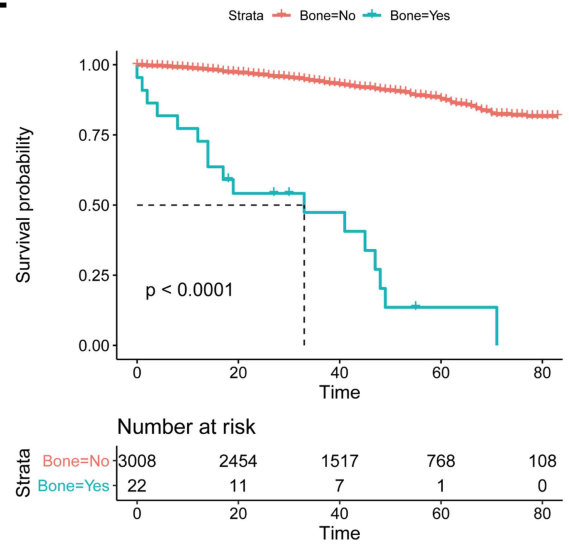

B

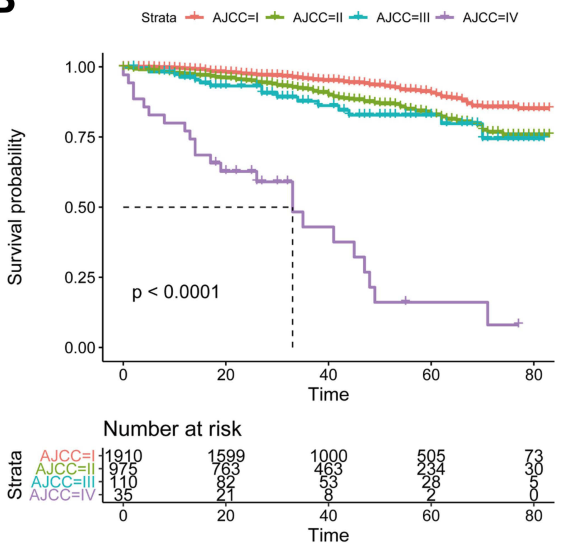

D

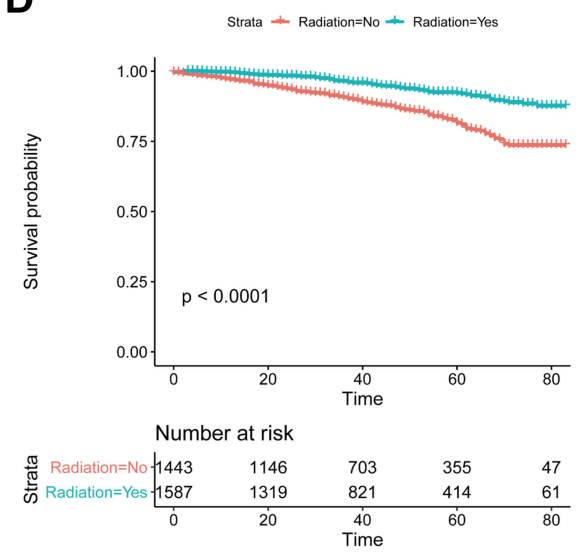

Figure I The K-M survival curves of each independent prognostic factor. (A) The K-M survival curves for age. (B) The K-M survival curves for AJCC. (C) The K-M survival curves for surgery. (D) The K-M survival curves for radiation. (E) The K-M survival curves for bone metastasis.

Abbreviations: K-M, Kaplan-Meier; AJCC, American Joint Committee on Cancer.

a subtype of breast cancer with a low local recurrence rate. Surgery is the primary treatment strategy. Among them, breast-conserving surgery (BCS) is the most commonly used surgical method, and the incidence of postoperative dysfunction is relatively low. ${ }^{28}$ The prognostic value of postoperative radiotherapy for MCB patients has been controversial. $^{29}$ Many previous studies indicated that increasing postoperative radiotherapy did not significantly improve OS. ${ }^{30,31}$ Conversely, Wu et $\mathrm{al}^{29}$ indicated that postoperative radiotherapy should not be omitted. 


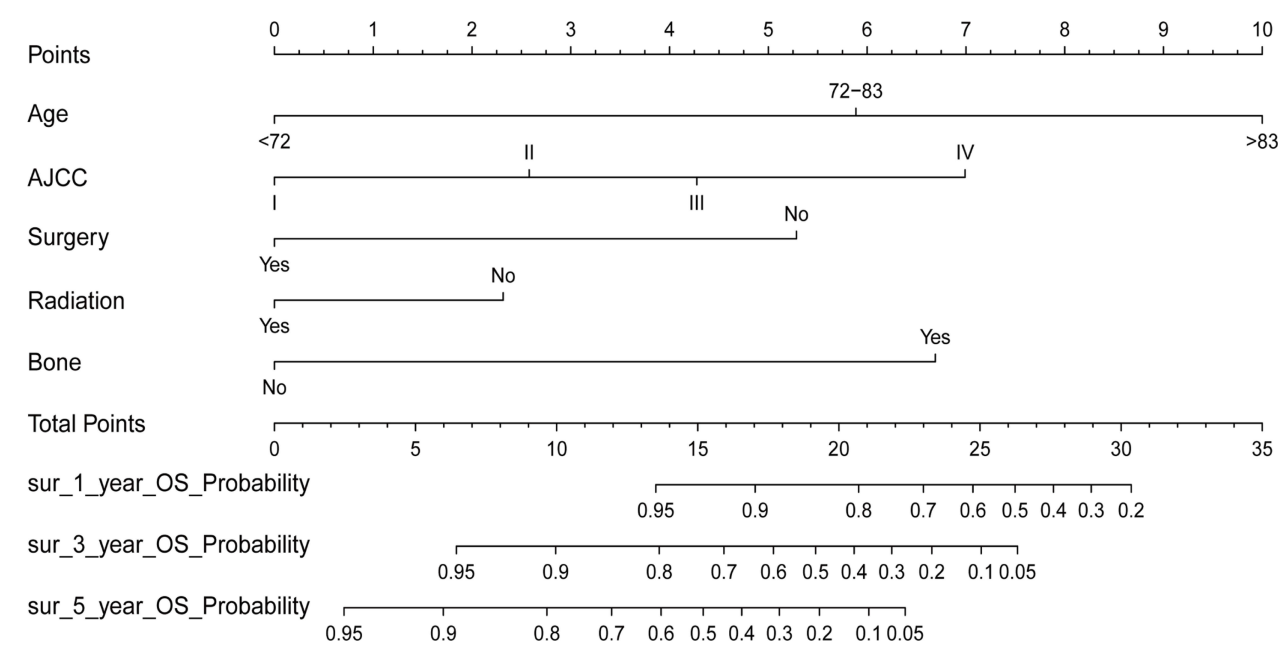

Figure 2 A nomogram for predicting the 3-, 4-, and 5-year overall survival rate for MCB patients. Abbreviation: MCB, mucinous carcinoma of the breast.
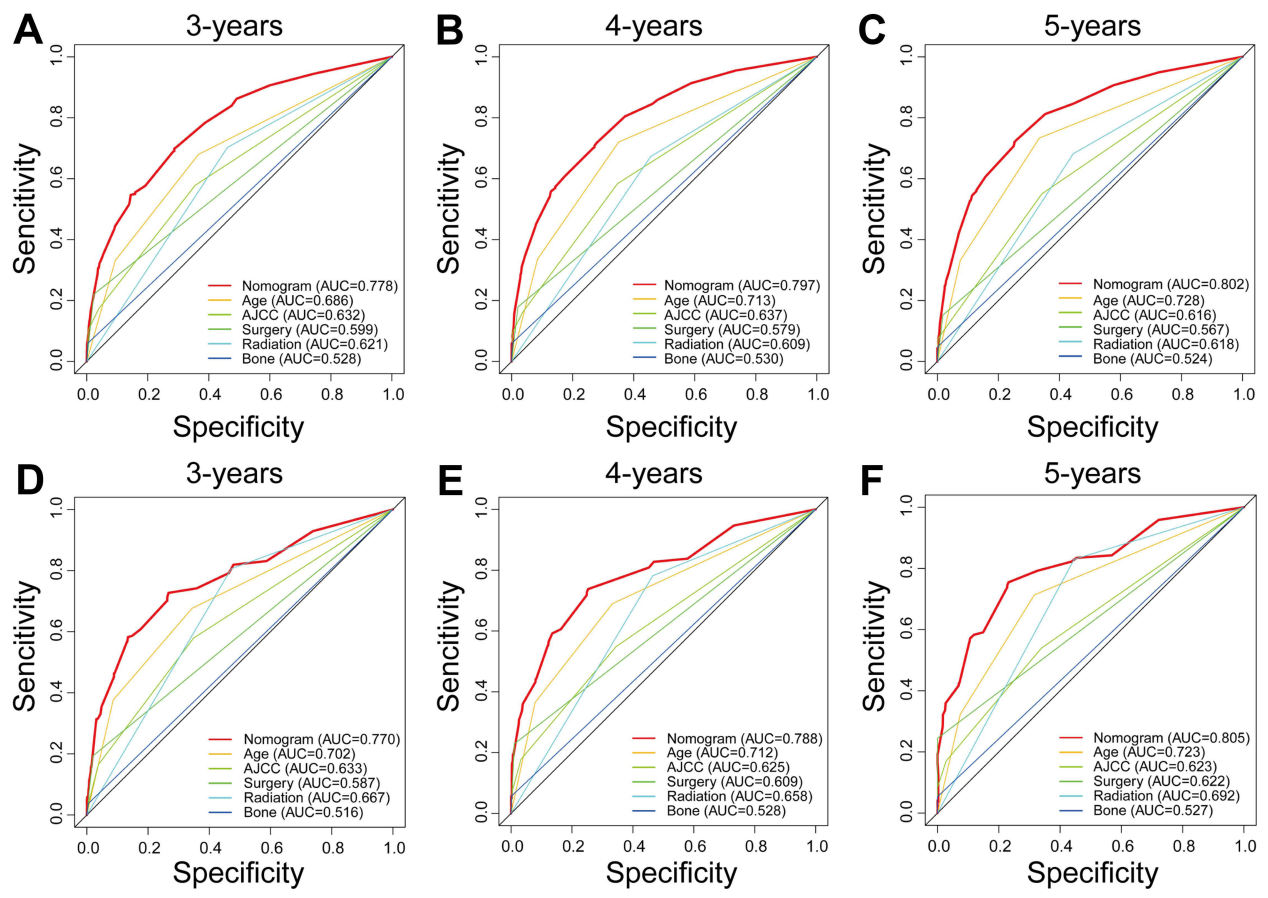

Figure 3 The ROC curves of the nomogram and all independent predictors at 3- (A), 4- (B), and 5-years (C) in the training set and at 3- (D), 4- (E), and 5-years (F) in testing set.

Abbreviation: ROC, receiver operating characteristic.

Although the MCB patients who received radiotherapy in our study showed a better prognosis, further research is needed to confirm.

However, there are several limitations to our work. First, this was a retrospective study with a large sample, so potential selection bias was inevitable. Secondly, the SEER database lacks endocrine therapy, chemotherapy regimens, radiotherapy doses, local recurrence and distant recurrence. Thirdly, the construction and validation of the prognostic nomogram were carried out in a single institution, which may affect its clinical application to a certain extent. Therefore, it is necessary to calibrate the nomogram in the future further.

\section{Conclusion}

In conclusion, routine clinical data were used to construct and validate the nomogram of MCB patients' outcomes at 

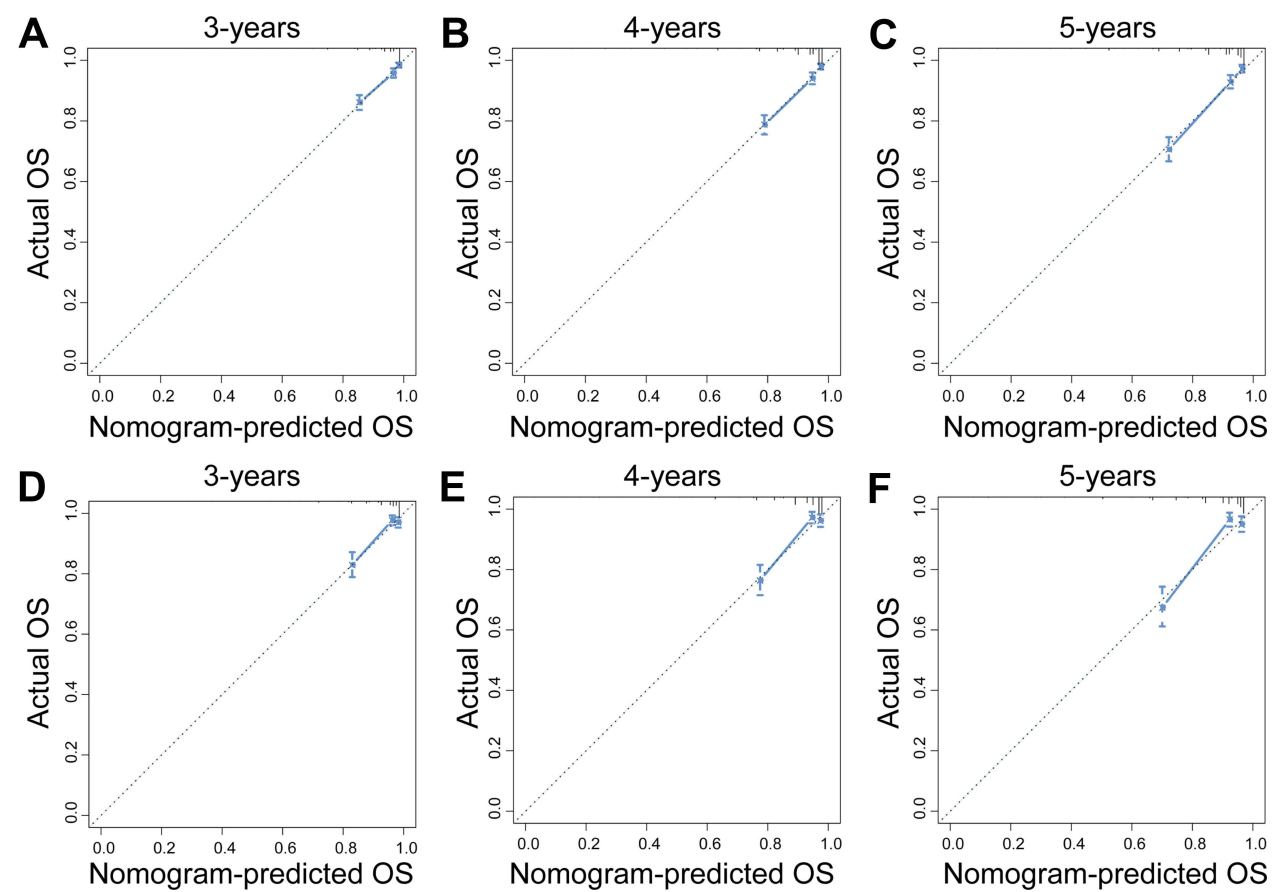

Figure 4 The calibration curves of the nomogram at 3- (A), 4- (B), and 5-years (C) in the training set and at 3- (D), 4- (E), and 5-years (F) in testing set.
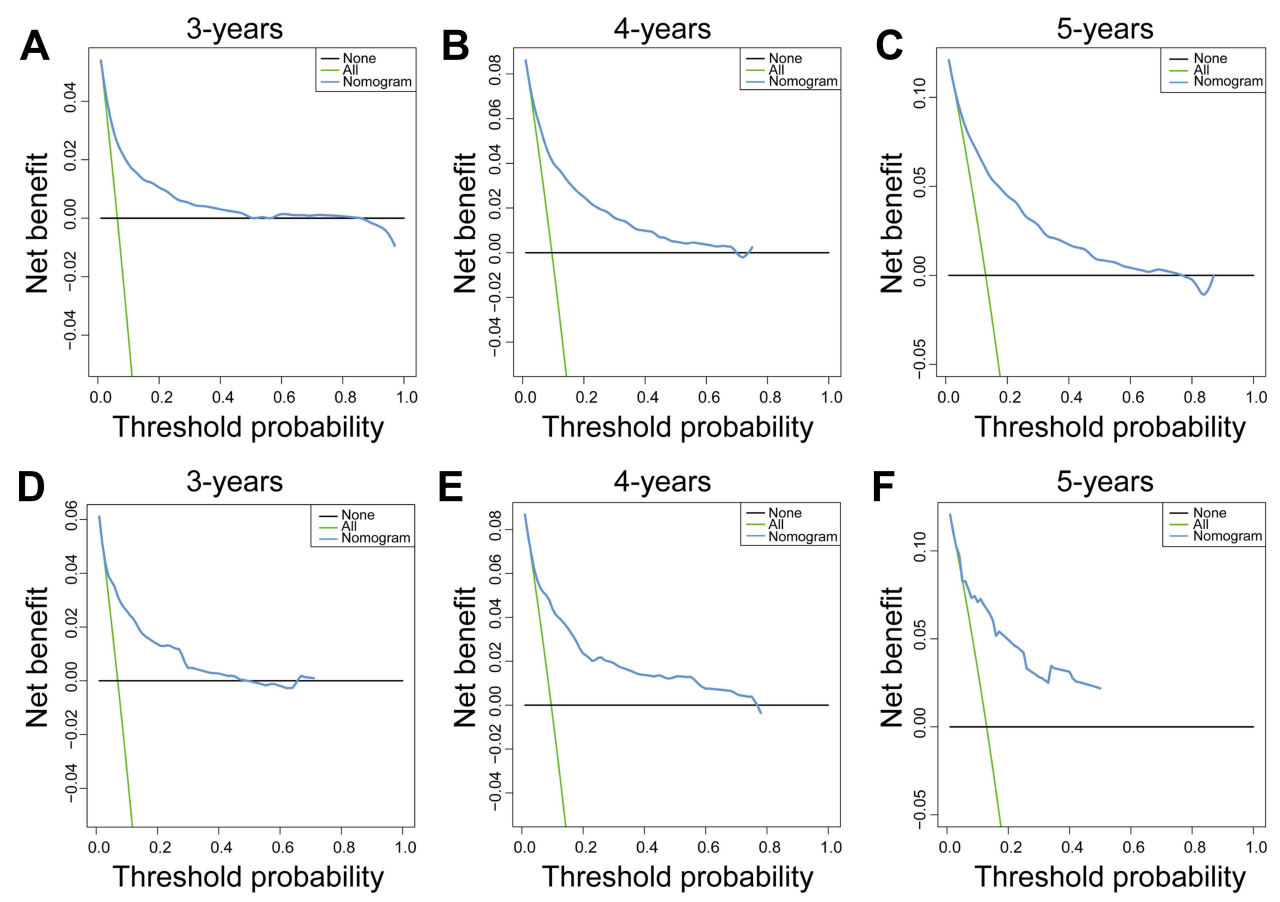

Figure 5 The DCA of the nomogram at 3- (A), 4- (B), and 5-years (C) in the training set and at 3- (D), 4- (E), and 5-years (F) in testing set. Abbreviation: DCA, decision curve analysis.

3-, 4-, and 5- years. Besides, the nomogram scoring systems had better discriminative power and clinical application value than the prognostic factors alone. Meanwhile, patients are classified into low-risk, middle-risk, and highrisk groups. This is very useful for promoting individualized therapy and management of MCB patients. 

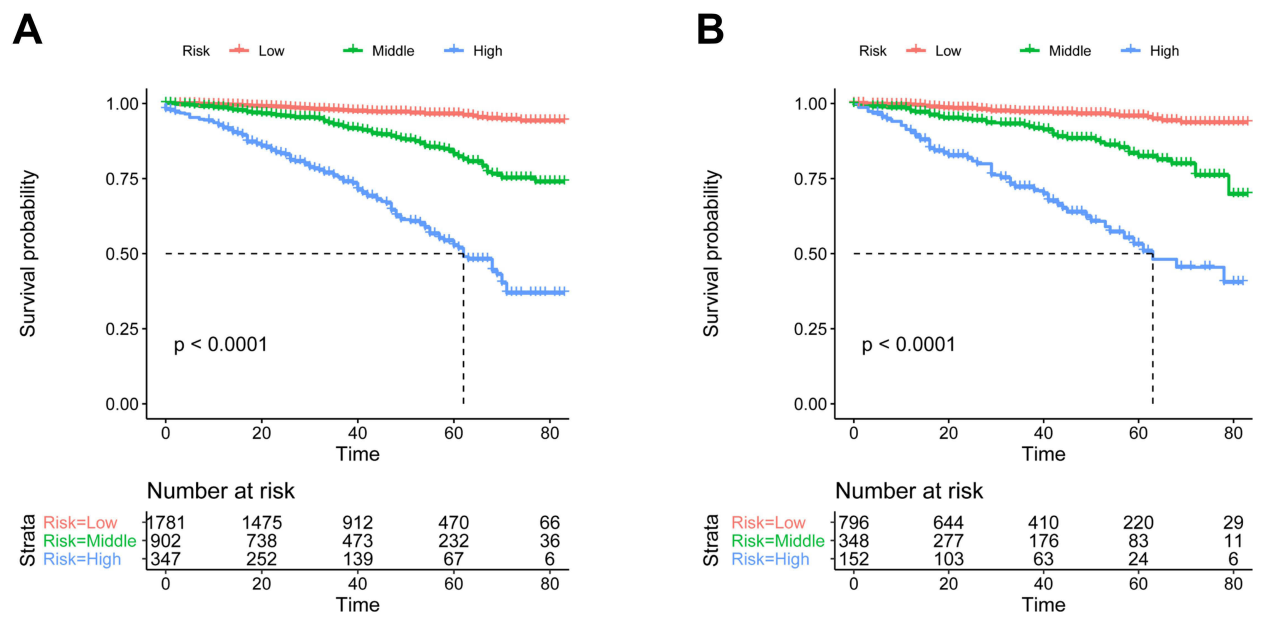

Figure 6 The K-M survival curves of three risk groups in the training set (A) and testing set (B).

Abbreviation: K-M, Kaplan-Meier.

\section{Abbreviations}

MCB, Mucinous carcinoma of the breast; SEER, Surveillance, Epidemiology, and End Result; K-M, Kaplan-Meier; ROC, receiver operating characteristic; DCA, decision curve analysis; AJCC, American Joint Committee on Cancer; AUCs, areas under the ROC curves; IDC, infiltrating ductal carcinoma; ER, estrogen receptor; PR, progesterone receptor; HER2, human epidermal growth factor 2-neu; BCS, breast-conserving surgery.

\section{Ethics Statement}

SEER belongs to public databases. The patients involved in the database have obtained ethical approval. Users can download relevant data for free for research and publish relevant articles. Our study is based on open source data, so there are no ethical issues.

\section{Ethics Approval and Informed Consent}

All information from the SEER program is available and free for public and approval was obtained from the Ethics Committee of First Affiliated Hospital of Wenzhou Medical University.

\section{Acknowledgments}

The authors thank the National Cancer Institute for providing the SEER data.

\section{Author Contributions}

All authors contributed to study design, acquisition, and analysis of data, and writing and revising manuscripts and reached a consensus on the journal to which the article was submitted, approved the final version to be published and agreed to be responsible for all content of the article.

\section{Funding}

This work was supported by the Ningxia Natural Science Foundation Project 2021 [grant number 2021AAC03523].

\section{Disclosure}

The authors report no conflicts of interest in this work.

\section{References}

1. Bray F, Ferlay J, Soerjomataram I, Siegel RL, Torre LA, Jemal A. Global cancer statistics 2018: GLOBOCAN estimates of incidence and mortality worldwide for 36 cancers in 185 countries. CA Cancer J Clin. 2018;68(6):394-424. doi:10.3322/caac.21492

2. Budzik MP, Fudalej MM, Badowska-Kozakiewicz AM. Histopathological analysis of mucinous breast cancer subtypes and comparison with invasive carcinoma of no special type. Sci Rep. 2021;11(1):5770. doi:10.1038/s41598-021-85309-z

3. Yu P, Liu P, Zou Y, et al. Breast-conserving therapy shows better prognosis in mucinous breast carcinoma compared with mastectomy: a SEER population-based study. Cancer Med. 2020;9(15):5381-5391. doi:10.1002/cam4.3202

4. Guo R, Chen B, Erickson LA. Mucinous carcinoma of the breast. Mayo Clin Proc. 2020;95(9):2049-2050. doi:10.1016/j.mayocp.2020.07.008

5. Harrison BT, Dillon DA. An update of mucinous lesions of the breast. Surg Pathol Clin. 2018;11(1):61-90. doi:10.1016/j.path.2017.09.002

6. Risner V, Jordan S. Mucinous carcinoma in an octogenarian: treatment and management of breast cancer in the elderly. Radiol Case Rep. 2021;16(11):3201-3207. doi:10.1016/j.radcr.2021.07.077

7. Limaiem F, Ahmad F. Mucinous Breast Carcinoma. Treasure Island (FL): StatPearls; 2021.

8. Alothman S, Saeed S, Khan K, Ramcharan A, DePaz H. Giant mucinous carcinoma of the breast. Cureus. 2018;10(11):e3606. doi: $10.7759 /$ cureus. 3606

9. Rasmussen BB, Rose C, Hilkens J, Hilgers J. Detection of surface antigens defined by monoclonal antibodies in primary mucinous breast carcinomas. Relation to prognostic factors and recurrence-free survival. Virchows Arch a Pathol Anat Histopathol. 1986;409 (4):497-505. doi:10.1007/BF00705420 
10. Skotnicki P, Sas-Korczynska B, Strzepek L, et al. Pure and mixed mucinous carcinoma of the breast: a comparison of clinical outcomes and treatment results. Breast J. 2016;22(5):529-534. doi:10.1111/ tbj. 12621

11. Amin MB, Greene FL, Edge SB, et al. The eighth edition AJCC cancer staging manual: continuing to build a bridge from a population-based to a more "personalized" approach to cancer staging. CA Cancer J Clin. 2017;67(2):93-99. doi:10.3322/ caac. 21388

12. Xu X, Bi R, Shui R, et al. Micropapillary pattern in pure mucinous carcinoma of the breast - does it matter or not? Histopathology. 2019;74(2):248-255. doi:10.1111/his.13722

13. Di Saverio S, Gutierrez J, Avisar E. A retrospective review with long term follow up of 11,400 cases of pure mucinous breast carcinoma. Breast Cancer Res Treat. 2008;111(3):541-547. doi:10.1007/s10549007-9809-z

14. Balachandran VP, Gonen M, Smith JJ, DeMatteo RP. Nomograms in oncology: more than meets the eye. Lancet Oncol. 2015;16(4):e173e180. doi:10.1016/S1470-2045(14)71116-7

15. Camp RL, Dolled-Filhart M, Rimm DL. X-tile: a new bio-informatics tool for biomarker assessment and outcome-based cut-point optimization. Clin Cancer Res. 2004;10(21):7252-7259. doi:10.1158/1078-0432.CCR-04-0713

16. Heagerty PJ, Lumley T, Pepe MS. Time-dependent ROC curves for censored survival data and a diagnostic marker. Biometrics. 2000;56 (2):337-344. doi:10.1111/j.0006-341X.2000.00337.x

17. Zhang L, Jia N, Han L, Yang L, Xu W, Chen W. Comparative analysis of imaging and pathology features of mucinous carcinoma of the breast. Clin Breast Cancer. 2015;15(2):e147-e154. doi:10.1016/j.clbc.2014.11.005

18. de Boer AZ, Bastiaannet E, Putter H, et al. Prediction of other-cause mortality in older patients with breast cancer using comorbidity. Cancers. 2021;13(7):1627.

19. Bergman L, Dekker G, van Kerkhoff EH, Peterse HL, van Dongen JA, van Leeuwen FE. Influence of age and comorbidity on treatment choice and survival in elderly patients with breast cancer Breast Cancer Res Treat. 1991;18(3):189-198. doi:10.1007/ BF01990035

20. Turashvili G, Brogi E, Morrow M, et al. The 21-gene recurrence score in special histologic subtypes of breast cancer with favorable prognosis. Breast Cancer Res Treat. 2017;165(1):65-76. doi:10.1007/ s10549-017-4326-1
21. Zulauf N, Bruggmann D, Groneberg D, Oremek GM. Expressiveness of bone markers in breast cancer with bone metastases. Oncology. 2019;97(4):236-244. doi:10.1159/000500675

22. He F, Chiou AE, Loh HC, et al. Multiscale characterization of the mineral phase at skeletal sites of breast cancer metastasis. Proc Natl Acad Sci U S A. 2017;114(40):10542-10547. doi:10.1073/ pnas. 1708161114

23. Louwman MWJ, Vriezen M, van Beek MWPM, et al. Uncommon breast tumors in perspective: incidence, treatment and survival in the Netherlands. Int $J$ Cancer. 2007;121(1):127-135. doi:10.1002/ ijc. 22625

24. Andre S, Cunha F, Bernardo M, Meneses e Sousa J, Cortez F, Soares J. Mucinous carcinoma of the breast: a pathologic study of 82 cases. J Surg Oncol. 1995;58(3):162-167. doi:10.1002/ jso. 2930580305

25. Koenders PG, Beex LV, Langens R, Kloppenborg PW, Smals AG, Benraad TJ. Steroid hormone receptor activity of primary human breast cancer and pattern of first metastasis. The Breast Cancer Study Group. Breast Cancer Res Treat. 1991;18(1):27-32. doi:10.1007/BF01975440

26. Coleman RE, Smith P, Rubens RD. Clinical course and prognostic factors following bone recurrence from breast cancer. $\mathrm{Br} J$ Cancer. 1998;77(2):336-340. doi:10.1038/bjc.1998.52

27. Solomayer EF, Diel IJ, Meyberg GC, Gollan C, Bastert G. Metastatic breast cancer: clinical course, prognosis and therapy related to the first site of metastasis. Breast Cancer Res Treat. 2000;59(3):271-278. doi:10.1023/A:1006308619659

28. Marrazzo E, Frusone F, Milana F, et al. Mucinous breast cancer: a narrative review of the literature and a retrospective tertiary single-centre analysis. Breast. 2020;49:87-92. doi:10.1016/j. breast.2019.11.002

29. Wu SG, Li FY, Wang J, Lian CL, Zhou J, He ZY. Omission of adjuvant radiotherapy following breast-conserving surgery for elderly women with early-stage pure mucinous breast carcinoma. Radiat Oncol. 2019;14(1):190. doi:10.1186/s13014-019-1394-x

30. Fu J, Wu L, Jiang M, et al. Clinical nomogram for predicting survival outcomes in early mucinous breast cancer. PLoS One. 2016;11(10): e0164921. doi:10.1371/journal.pone.0164921

31. Gwark SC, Lee HS, Lee Y, et al. Clinical implication of HER2 status in hormone receptor-positive mucinous breast cancer. Ann Surg Oncol. 2019;26(7):2166-2174. doi:10.1245/s10434-019-07332-9
International Journal of General Medicine

\section{Publish your work in this journal}

The International Journal of General Medicine is an international, peer-reviewed open-access journal that focuses on general and internal medicine, pathogenesis, epidemiology, diagnosis, monitoring and treatment protocols. The journal is characterized by the rapid reporting of reviews, original research and clinical studies across all disease areas. The manuscript management system is completely online and includes a very quick and fair peer-review system, which is all easy to use. Visit http://www.dovepress.com/ testimonials.php to read real quotes from published authors. 